\title{
On the Dynamic Optimization of Biped Robot
}

\author{
Hayder F. N. Al-Shuka, Member, IACSIT, Burkhard J. Corves, and Wen-Hong Zhu
}

\begin{abstract}
This paper concentrates on three important points: the selection of the suitable direct method used for suboptimal control of the biped robot, the selection of the appropriate nonlinear programming (NLP) algorithm that searches for the global minimum rather than the local minimum, and the effect of different constraints on the energy of the biped robot. To perform the mentioned points, the advantages and disadvantages of the optimal control methods were illustrated. The inverse-dynamics based optimization is preferred because of the ability to convert the original optimal control into algebraic equations which are easy to deal with. The inverse-dynamics-based optimization was classified as spline and the finite difference based optimization. Due to the easy use of the latter, it was used for investigating seven cases with different constraints for 6-DOF biped robot during the single support phase (SSP). Hybrid genetic-sequential quadratic programming (GA-SQP) was used for simulation of the target robot with MATLAB. It can be concluded that more imposed constraints on the biped robot, more energy is needed. In general, more energy can be required in the case of (1) restriction of the swing foot to be level to the ground and (2) reducing the hip height or constraining the hip to move in constant height.
\end{abstract}

Index Terms-Biped robot, dynamic optimization suboptimal control, walking patterns.

\section{INTRODUCTION}

One of the important issues of the biped locomotion is the generation of the desired paths that ensure stability and avoid collision with obstacles [1]. Numerous approaches have been used to generate the motion of the biped robot as detailed in [2]. However, there are two efficient methods used for this purpose: center of gravity (COG)-based gait and the optimization-based gait. The former deals with a simplified model assuming all the masses of the biped robot are concentrated in the COG of the biped and there is pushing force at the ankle support foot without ankle torque applied [3]. This method can guarantee the stability of the biped robot provided that full correction for the deviation of the zero-moment point is performed. However, it does not deal with the minimum energy, optimal design, and the different kinematic and dynamic constraints of the biped robot. The latter can be dealt successfully with the optimal control theory [4]. In general, the optimal control can be classified as: dynamic programming, indirect methods and

Manuscript received February 3, 2013; revised April 10, 2013. This work was supported in part by a grant from German Academic Exchange Service (DAAD) and the Ministry of Higher Education and Scientific Research of Iraq (MoHESR).

Hayder F. N. Al-Shuka and B. J. Corves are with Department of Mechanism and Machine Dynamics, Aachen, Germany (e-mail: hayder.alshuka@rwth-aachen.de)

Wen-Hong Zhu is with the Canadian Space Agency, Canada (e-mail: Wen-Hong.Zhu@asc-csa.gc.ca) direct methods as shown in Fig. 1. Although, the dynamic programming is less sensitive to the initial guess of the design parameters, it suffers from the curse of dimensionality [5]. The indirect approach represented by PMP demands necessary conditions for optimality, which results in nonlinear, two-boundary value problem [6], [7] However, the computational solution may lead to highly nonlinear ODEs. Obtaining necessary conditions of optimality can be intricate for complex dynamic systems such as biped robot [4], [8]. In addition, the indirect methods are extremely sensitive to the initial guess of the costate equations. Despite this difficulty, [9], [10] have investigated the optimal motion of the biped robot during single support phase (SSP) and during the complete gait cycle respectively using PMP assuming the boundary conditions of the biped robot are known.

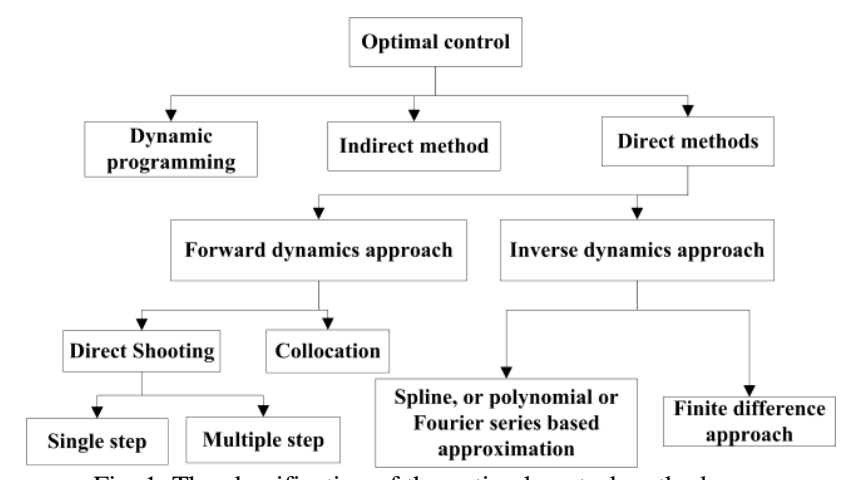

Fig. 1. The classification of the optimal control methods.

Therefore, the analyst needs more flexible methods for optimal control problems, represented by the direct methods, by transcribing the infinite dimension problem into finitedimensional nonlinear programming (static or parameter optimization). This can be implemented by discretization of the controls or the states or both of them, depending on the selected discretization approach, and solving the problem using one of the nonlinear programming algorithms such as sequential quadratic programming (SQP), interior points, genetic algorithm (GA) etc. Although its ease and robustness, this method can only give suboptimal/approximate solution [6], [7], [11], [12].

This paper concentrates on three important points: selection of the suitable direct method used for suboptimal control of the biped robot, selection of the appropriate nonlinear programming (NLP) algorithm that searches for the global minimum rather than the local minimum, and the effect of different constraints on the energy of the biped robot.

The structure of the paper is as follows. A review of the suboptimal control for the mechanical systems is introduced in Section II. Section III investigates the dynamic optimization of the biped robot based on the finite difference 
approach. While Section IV shows the simulation results and discussions. The conclusion is considered in Section V.

\section{REVIEW OF THE SUBOPTIMAL CONTROL FOR THE MECHANICAL SYSTEMS}

\section{A. Forward-Dynamics Based Optimization}

The formulation of the original optimal control problem can be described as follows:

\section{Determine: $\boldsymbol{u}$}

Minimize: $J=c_{0}(\boldsymbol{x}, t)+\int_{t_{0}}^{t_{f}} L(\boldsymbol{x}(t), \boldsymbol{u}(t), t) d t$

Subject to:

$$
\begin{gathered}
\dot{x}=\boldsymbol{f}(\boldsymbol{x}(t), \boldsymbol{u}(t), t) \\
\boldsymbol{a}_{1}\left(\boldsymbol{x}\left(t_{0}\right), \boldsymbol{u}\left(t_{0}\right), t_{0}\right) \leq 0 \\
\boldsymbol{a}_{\mathbf{2}}\left(\boldsymbol{x}\left(t_{0}\right), \boldsymbol{u}\left(t_{0}\right), t_{0}\right)=0 \\
\boldsymbol{b}_{1}\left(\boldsymbol{x}\left(t_{f}\right), \boldsymbol{u}\left(t_{f}\right), t_{f}\right) \leq 0 \\
\boldsymbol{b}_{\mathbf{2}}\left(\boldsymbol{x}\left(t_{f}\right), \boldsymbol{u}\left(t_{f}\right), t_{f}\right)=0 \\
\boldsymbol{c}_{1}(\boldsymbol{x}(t), \boldsymbol{u}(t), t) \leq 0 \\
\boldsymbol{c}_{\mathbf{2}}(\boldsymbol{x}(t), \boldsymbol{u}(t), t)=0 \\
\boldsymbol{u}_{\boldsymbol{l}} \leq \boldsymbol{u}(t) \leq \boldsymbol{u}_{\boldsymbol{u}}
\end{gathered}
$$

TABLE I: The Formulations, AdVANTAges and Disadvantages of the Single Shooting AND the Collocation Methods Single-shooting [7], [13], [14]
Principle:

Discretizing the control variables only.

Suboptimal problem:

Determine : $\boldsymbol{Y}=\left[u\left(t_{0}\right), \ldots, u\left(t_{N}\right)\right]$

Minimize : Equation (7)

Subject to:

$\boldsymbol{x}_{k+1}=\boldsymbol{x}_{k}+\Delta t . \varphi, k=0, \ldots, N-1$

$\boldsymbol{a}_{\mathbf{1}}\left(\boldsymbol{x}\left(t_{0}\right), \boldsymbol{u}\left(t_{0}\right), t_{0}\right) \leq \mathbf{0}$

$\boldsymbol{a}_{2}\left(\boldsymbol{x}\left(t_{0}\right), \boldsymbol{u}\left(t_{0}\right), t_{0}\right)=\mathbf{0}$

$\boldsymbol{b}_{1}\left(\boldsymbol{x}\left(t_{N}\right), \boldsymbol{u}\left(t_{N}\right), t_{N}\right) \leq \mathbf{0}$

$\boldsymbol{b}_{2}\left(\boldsymbol{x}\left(t_{N}\right), \boldsymbol{u}\left(t_{N}\right), t_{N}\right)=\mathbf{0}$

$\boldsymbol{c}_{\mathbf{1}}\left(\boldsymbol{x}\left(t_{k}\right), \boldsymbol{u}\left(t_{k}\right), t_{k}\right) \leq \mathbf{0}, \quad k=0, \ldots, N$

$\boldsymbol{c}_{2}\left(x\left(t_{k}\right), \boldsymbol{u}\left(t_{k}\right), t_{k}\right)=\mathbf{0}, k=0, \ldots, N$

$\boldsymbol{u}_{\boldsymbol{l}} \leq \boldsymbol{u}\left(t_{k}\right) \leq \boldsymbol{u}_{\boldsymbol{u}}, k=0, \ldots, N$

$\boldsymbol{x}_{\boldsymbol{l}} \leq \boldsymbol{x}\left(t_{k}\right) \leq \boldsymbol{x}_{\boldsymbol{u}}, k=0, \ldots, N$

where $\varphi$ is the slope which can adopt different formulae resulting in Euler, Heun, Mid-point, and Runge-Kutta methods.

Advantages :

- It has few design variables even for large scale systems

- Equation (12) can be solved by any ODE solver such as Euler, Mid-point, Heun and Runge-Kutta methods.

Disadvantages:

- It cannot use knowledge of state vector $\mathrm{x}$ in initialization.

- The state solution can depend nonlinearly on the discrete control vector.

- It is not preferred for unstable system

$$
x_{\boldsymbol{l}} \leq \boldsymbol{x}(t) \leq \boldsymbol{x}_{\boldsymbol{u}}
$$

where $\boldsymbol{u} \in \mathbb{R}^{n}$ is the input control vector, $c_{0}$ and $L$ are scalar functions of the indicated arguments, $J$ is a scalar performance index, $\boldsymbol{x} \in \mathbb{R}^{n}$ is the state vector, $t, t_{0}$ and $t_{f}$ are the time, initial and final time respectively, $\boldsymbol{a}_{\mathbf{1}}$ and $\boldsymbol{a}_{\mathbf{2}}$ are the initial constraints, $\boldsymbol{b}_{\mathbf{1}}$ and $\boldsymbol{b}_{\mathbf{2}}$ are the final constraints, $\boldsymbol{c}_{1}$ and $\boldsymbol{c}_{2}$ are the path constrains and (6) refers to the bound constraints of the input control and the states.

The formulation of discretized optimal control problem can be described as a nonlinear programming as follows:

Determine: $\boldsymbol{Y}$ which it may be the control variables or the states or both of them.

Minimize:

$$
J=c_{0}\left(\boldsymbol{x}\left(t_{N}\right)\right)+\sum_{k=0}^{N-1} l_{k}\left(\boldsymbol{x}\left(t_{k}\right), \boldsymbol{u}\left(t_{k}\right), t_{k}\right) \Delta t
$$

where $N$ is the number of the time intervals and $\Delta t=\left(t_{f}-\right.$ $\left.t_{0}\right) / N$. Equation (7) can be solved by any numerical integration approach such as trapezoidal or composite Simpson's rule etc.

Subject to:

$$
\begin{gathered}
\boldsymbol{Z}(\boldsymbol{Y})=0 \\
\boldsymbol{C}_{\boldsymbol{l}} \leq \boldsymbol{C}(\boldsymbol{Y}) \leq \boldsymbol{C}_{\boldsymbol{u}} \\
\boldsymbol{Y}_{\boldsymbol{l}} \leq \boldsymbol{Y} \leq \boldsymbol{Y}_{\boldsymbol{u}}
\end{gathered}
$$

(11) Determine : $\boldsymbol{Y}=\left[u\left(t_{0}\right), \ldots, u\left(t_{N}\right), x\left(t_{0}\right), \ldots, x\left(t_{N}\right)\right]$

Minimize : Equation (7)

Subject to:

(12) $\boldsymbol{f}\left(\widetilde{\boldsymbol{x}}\left(t_{m, k}\right), \widetilde{\boldsymbol{u}}\left(t_{m, k}\right), t_{m, k}\right)-\dot{\tilde{\boldsymbol{x}}}\left(t_{m, k}\right)=\mathbf{0}, k=0, \ldots, N-1$

$\boldsymbol{a}_{\mathbf{1}}\left(\widetilde{\boldsymbol{x}}\left(t_{0}\right), \widetilde{\boldsymbol{u}}\left(t_{0}\right), t_{0}\right) \leq \mathbf{0}$

(13) $\quad \boldsymbol{a}_{2}\left(\widetilde{\boldsymbol{x}}\left(t_{0}\right), \widetilde{\boldsymbol{u}}\left(t_{0}\right), t_{0}\right)=\mathbf{0}$

$\boldsymbol{b}_{\mathbf{1}}\left(\widetilde{\boldsymbol{x}}\left(t_{N}\right), \widetilde{\boldsymbol{u}}\left(t_{N}\right), t_{N}\right) \leq \mathbf{0}$

(14) $\boldsymbol{b}_{\mathbf{2}}\left(\widetilde{\boldsymbol{x}}\left(t_{N}\right), \widetilde{\boldsymbol{u}}\left(t_{N}\right), t_{N}\right)=\mathbf{0}$

$\boldsymbol{c}_{\mathbf{1}}\left(\widetilde{\boldsymbol{x}}\left(t_{m, k}\right), \widetilde{\boldsymbol{u}}\left(t_{m, k}\right), t_{k}\right) \leq \mathbf{0}, \quad k=0, \ldots, N$

(15) $\quad \boldsymbol{c}_{2}\left(\widetilde{\boldsymbol{x}}\left(t_{m, k}\right), \widetilde{\boldsymbol{u}}\left(t_{m, k}\right), t_{k}\right)=\mathbf{0}, k=0, \ldots, N$

$\boldsymbol{u}_{\boldsymbol{l}} \leq \boldsymbol{u}\left(t_{k}\right) \leq \boldsymbol{u}_{\boldsymbol{u}}, k=0, \ldots, N$

$x_{l} \leq \boldsymbol{x}\left(t_{k}\right) \leq \boldsymbol{x}_{\boldsymbol{u}}, k=0, \ldots, N$

where

$t_{m, k}=\left(t_{k}+t_{k+1}\right) / 2 k=0, \ldots, N-1$

$\widetilde{\boldsymbol{x}}_{i}(t)=\sum_{i=0}^{3} c_{i}^{k}\left(\frac{t-t_{k}}{\Delta t}\right)^{i}, t_{k} \leq t \leq t_{k+1}, k=0, \ldots, N-1$

$\widetilde{\boldsymbol{u}}(t)=u\left(t_{k}\right)+\frac{t-t_{k}}{\Delta t}\left(u\left(t_{k+1}\right)-u\left(t_{k}\right)\right), t_{k} \leq t \leq t_{k+1}, k=$ $0, \ldots, N-1$

Advantages :

- $\quad$ The resulting solution is large scale system with sparse NLP.

- It can use the knowledge of the state vector in the initialization.

- It can cope with unstable system and different constraints reliably.

Disadvantages:

- It needs more computational time than single-shooting approach, due to large design parameters used. 
Table I shows the formulations, advantages and disadvantages of the single-shooting and the collocation methods. Multiple-shooting is a combination of these two methods and it is not considered here. For further details, we refer to [7], [11], [13].

Remark 1. When applying the forward-dynamics based optimization on the multi-body dynamics (robotic system), the following issues should be noticed:

- Equation (2) needs calculation of the inverse mass matrix of the robotic system which may result in computational difficulty.

- If the multibody dynamic systems move with constrained motion, the equality and inequality constraints may not have explicit expressions for the input control variables. Consequently, these constraints must be derived many times until the input control vector appears. For detail, we refer to [17].

- To solve the NLP, it is necessary to choose feasible initial guess for the design variables. Consequently, it is not easy to get a good initial guess for the control variables at the forward-dynamics based methods.

Despite the difficulties encountered in the solution of forward dynamics-based optimization, it is adopted as an optimization tool for generating optimal walking patterns of biped robot in [18], [19].

Remark 2. After converting the original optimal control problem into NLP, the routine fmincon of the MATLAB Optimization Toolbox can be used easily. In fact, most of the MATLAB routines can be used effectively: $g a$ (genetic algorithm), GlobalSearch, Multistart and the PatternSearch [20], [21]. V. M. Becerra [15] has given a simple detailed example of fmincon to solve the collocation approach.

\section{B. Inverse-Dynamics Based Optimization}

The difference between the inverse-dynamics and forward-dynamics based optimization appears in the formulation of (2), such that the dynamics equation for the target system of the inverse-dynamics approach is not written in the state space form. Therefore, this approach has three distinctive features: (1) it does not need the inverse mass matrix, (2) only the states of the target system are discretized, and (3) the ability to convert the original optimal control into algebraic equations which are easy to deal with.

For multibody system (robotic system), the dynamics equation can be written in a standard Lagrangian equation as follows

$$
M \ddot{q}+C \dot{q}+g=A \tau
$$

where $\boldsymbol{M} \in \mathbb{R}^{n \times n}$ is the mass robot matrix, $n$ denotes the DOF of the target robot, $\boldsymbol{q}, \dot{\boldsymbol{q}}$ and $\ddot{\boldsymbol{q}} \in \mathbb{R}^{n}$, are the absolute angular displacement, velocity and acceleration of the robot links, $\boldsymbol{C} \in \mathbb{R}^{n \times n}$ represents the Coriolis robot matrix, $\boldsymbol{g} \in \mathbb{R}^{n}$ is the gravity vector, $\boldsymbol{A} \in \mathbb{R}^{n \times n}$ is a mapping matrix derived by the principle of the virtual work [22], [23], and $\boldsymbol{\tau} \in \mathbb{R}^{n}$ is the actuating torque vector. This equation is valid for open-chain robotic system. For closed-chain mechanism, the Lagrangian multipliers should appear to right side of (26). In general, our current study will focus on the SSP of the biped robot.

L. Roussel et al [24] have made a comparative study on the dynamic optimization of point-feet biped robot. The authors have considered the forward dynamics approach using the single-shooting approach with the Euler method as integration method, and the inverse-dynamic approach using the polynomial approximation and the combined polynomial-Fourier series which is used by [25]. They have not considered the piecewise spline and the finite difference based optimization.

Spline-based optimization has been used extensively in the literatures [8], [22], [26], [27]. The first reference has used piecewise fourth-order spline function because the cubic spline functions may result in discontinuities in the third derivative of the approximated joint displacements. However, literatures have approved the efficiency of the cubic-spline functions in the approximation. In our paper, we consider two efficient tools for the solution of inversedynamics approach: the piecewise cubic spline functions and the finite difference equations.

A detailed study on the spline based optimization of the biped robot can be found in [8]. In the spline-based optimization, the angular link displacements of the robotic system are discretized into equal segments $(\mathrm{N})$, and it can be approximated as

$$
\widetilde{\boldsymbol{q}}_{k}(t)=\sum_{i=0}^{3} c_{i}^{k}\left(\frac{t-t_{k}}{\Delta t}\right)^{i}, t_{k} \leq t \leq t_{k+1}, k=0, \ldots, N-1
$$

Thus, we have a piecewise spline function for every interval (segment) with four coefficients to be determined using the following connecting and boundary conditions:

- At the intermediate connecting grids

$$
\begin{gathered}
\widetilde{\boldsymbol{q}}_{k-1}(1)=\boldsymbol{q}\left(t_{k}\right), \widetilde{\boldsymbol{q}}_{k}(0)=\boldsymbol{q}\left(t_{k}\right) \\
\dot{\widetilde{\boldsymbol{q}}}_{k-1}(1)=\dot{\widetilde{\boldsymbol{q}}}_{k}(0), \dot{\widetilde{\boldsymbol{q}}}_{k-1}(1)=\dot{\widetilde{\boldsymbol{q}}}_{k}(0), k=1, \ldots, N-1
\end{gathered}
$$

- At the boundary conditions

$$
\begin{gathered}
\widetilde{\boldsymbol{q}}_{1}(0)=\dot{\boldsymbol{q}}\left(t_{0}\right), \dot{\widetilde{\boldsymbol{q}}}_{1}(0)=\dot{\boldsymbol{q}}\left(t_{0}\right), \widetilde{\boldsymbol{q}}_{N}(1)=\boldsymbol{q}\left(t_{N}\right) \\
\dot{\widetilde{\boldsymbol{q}}}_{N}(1)=\dot{\boldsymbol{q}}\left(t_{N}\right)
\end{gathered}
$$

All the coefficients of the piecewise spline functions can be found if the displacements of the robotic system are known at the grids and the derivatives of these displacements are known at the boundary conditions. Consequently, the design parameters that should be optimized are the displacements of the grid points as well as their derivatives at the boundary conditions only.

The formulation of the piecewise spline-based optimization can be described as

Determine: $\boldsymbol{Y}=\left[\boldsymbol{q}\left(t_{0}\right), \ldots, \boldsymbol{q}\left(t_{N}\right), \dot{\boldsymbol{q}}\left(t_{0}\right), \dot{\boldsymbol{q}}\left(t_{N}\right)\right]$

Minimize: Equation (7)

Subject to:

$$
\begin{gathered}
\boldsymbol{M}\left(\widetilde{\boldsymbol{q}}_{k}\left(t_{k}\right)\right) \ddot{\tilde{\boldsymbol{q}}}_{k}\left(t_{k}\right)+\boldsymbol{C}\left(\widetilde{\boldsymbol{q}}_{k}\left(t_{k}\right), \dot{\widetilde{\boldsymbol{q}}}_{k}\left(t_{k}\right)\right) \dot{\widetilde{\boldsymbol{q}}}_{k}\left(t_{k}\right)+ \\
\boldsymbol{g}\left(\widetilde{\boldsymbol{q}}_{k}\left(t_{k}\right)\right)=\boldsymbol{A} \boldsymbol{\tau}\left(t_{k}\right), \quad k=0, \ldots, N
\end{gathered}
$$


with (13)-(16).

Whereas in the finite difference approach, the velocity and acceleration of the dynamic system can be approximated directly using the finite difference approach as follows.

$\dot{\boldsymbol{q}}\left(t_{k}\right)=\left(\boldsymbol{q}\left(t_{k+1}\right)-\boldsymbol{q}\left(t_{k-1}\right)\right) / 2 . \Delta t, k=0, \ldots, N$

$\ddot{\boldsymbol{q}}\left(t_{k}\right)=\left(\boldsymbol{q}\left(t_{k+1}\right)-2 \boldsymbol{q}\left(t_{k}\right)+\boldsymbol{q}\left(t_{k-1}\right)\right) / \Delta t^{2}, k=0, \ldots, N$

For detail we refer to [28], [29]. Thus, the formulation of the finite difference based optimization is as follows:

Determine: Equation (30)

Minimize: Equation (7)

subject to: $\boldsymbol{M}\left(\boldsymbol{q}\left(t_{k}\right)\right) \ddot{\boldsymbol{q}}\left(t_{k}\right)+\boldsymbol{C}\left(\boldsymbol{q}\left(t_{k}\right), \dot{\boldsymbol{q}}\left(t_{k}\right)\right) \dot{\boldsymbol{q}}\left(t_{k}\right)+$ $\boldsymbol{g}\left(\boldsymbol{q}\left(t_{k}\right)\right)=\boldsymbol{A} \boldsymbol{\tau}\left(t_{k}\right), \quad k=0, \ldots, N$

with (13) to (16).

Remark 3. In effect, we applied the spline-based optimization and the finite difference approach on a simple example cited from [6]. The results show that the latter can be implemented faster and easier than the former. We will not describe the details here due to the limited space.

\section{DYNAMIC OPTIMIZATION OF 6-DOF BIPED ROBOT}

Due to the easy use of the finite difference-based optimization, it was employed to generate optimal motion of the 6-DOFs biped robot during the single support phase (SSP) with different cases as we will see in the simulation section. Fig.2a shows the structure of the investigated biped robot. The physical parameters are shown in TABLE II. This section identifies the necessary constraints that can guarantee natural human motion, as follows:

1) Boundary conditions constraints

- Initial configuration of the swing leg:

$$
\begin{aligned}
x_{s A}\left(t_{0}\right)+L_{\text {step }}= & 0, \dot{x}_{s A}\left(t_{0}\right)=0, y_{s A}\left(t_{0}\right)=0, \\
& \dot{y}_{s A}\left(t_{0}\right)=0
\end{aligned}
$$

- Final configuration of the swing leg:

$$
\begin{gathered}
x_{s B}\left(t_{N}\right)-L_{\text {step }}=0, \dot{x}_{s B}\left(t_{N}\right)=0, y_{s B}\left(t_{N}\right)=0, \\
\dot{y}_{s B}\left(t_{N}\right)=0
\end{gathered}
$$

where all the notations are shown in Fig. 2.

2) Path constraints

- Hip motion:

$$
\begin{gathered}
\dot{x}_{\text {hip }}\left(t_{k}\right)>0, k=0, \ldots, N \\
h_{\text {min }} \leq y_{\text {hip }}\left(t_{k}\right) \leq h_{\text {max }}, k=0, \ldots, N
\end{gathered}
$$

- Swing foot motion:

$$
\begin{aligned}
& 0.01 \leq y_{s A}\left(t_{k}\right), k=1, \ldots, N-1 \\
& 0.01 \leq y_{s B}\left(t_{k}\right), k=1, \ldots, N-1
\end{aligned}
$$

- Relative displacement of the knee joints and swing ankle:

$$
\begin{aligned}
5 & \leq q_{2}\left(t_{k}\right)-q_{1}\left(t_{k}\right) \leq p i / 2, k=0, \ldots, N \\
5 & \leq q_{4}\left(t_{k}\right)-q_{5}\left(t_{k}\right) \leq p i / 2, k=0, \ldots, N \\
p i / 2 & \leq q_{6}\left(t_{k}\right)-q_{5}\left(t_{k}\right) \leq 3 p i / 2, k=0, \ldots, N
\end{aligned}
$$

- ZMP-constraint:

In general, there are two concepts used in the literature as follows:

Concept 1. This is commonly used in the field of biped robot which states that the location of reaction force is equal to the ZMP as long as the biped mechanism is stable, as shown in Fig. 2(b), therefore;

$$
\begin{gathered}
u_{1}\left(t_{k}\right)+F_{y}\left(t_{k}\right) x_{Z M P}\left(t_{k}\right)-m_{6} g d_{6}=0 \\
x_{Z M P}\left(t_{k}\right)=\left(m_{6} g d_{6}-u_{1}\left(t_{k}\right)\right) / F_{y}\left(t_{k}\right), k=0, \ldots, N
\end{gathered}
$$

Thus, the necessary associated constraint is

$$
-l_{f 2} \leq x_{Z M P}\left(t_{k}\right) \leq l_{f 1}, k=0, \ldots, N
$$

where $d_{6}=\overline{O_{6} C_{6}}, l_{f 1}=\overline{O_{6} A}$ and $l_{f 1}=\overline{O_{6} B}$

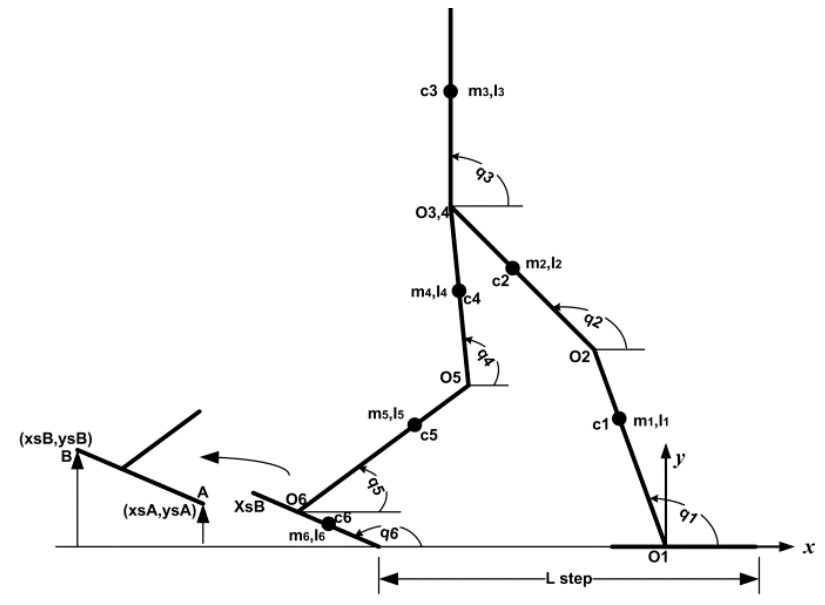

(a)
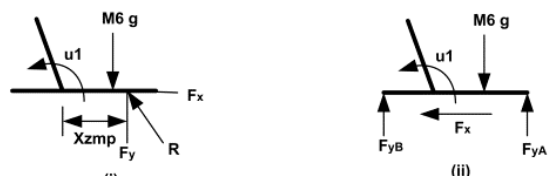

(b)

Fig. 2. The biped robot configuration at the initial single support phase.

\begin{tabular}{cllll}
\multicolumn{6}{c}{ TABLE II: THE PHYSICAL PARAMETERS OF THE BIPED ROBOT [30] } \\
\hline \hline$i$ & \multicolumn{1}{c}{$l_{i}=\overline{O_{i} O_{i+1}}$} & \multicolumn{1}{c}{$d_{i}=\overline{O_{i} c_{i}}$} & \multicolumn{1}{c}{$m_{i}$} & \multicolumn{1}{c}{$I_{i}$} \\
\hline 1 & 0.45 & 0.26 & 3.61 & 0.06 \\
2 & 0.45 & 0.261 & 3.69 & 0.062 \\
3 & 0.45 & 0.2 & 10.3 & 0.145 \\
4 & 0.45 & 0.189 & 3.66 & 0.06 \\
5 & 0.45 & 0.192 & 3.53 & 0.058 \\
6 & 0.3 & 0.073 & 2.05 & 0.016 \\
\hline \hline
\end{tabular}

In addition, the following constraints of the reaction forces that should be satisfied 


$$
\begin{gathered}
-F_{y}\left(t_{k}\right)<0, k=0, \ldots, N \\
-F_{x}-\mu F_{y}\left(t_{k}\right)<0, \quad k=0, \ldots, N \\
F_{x}-\mu F_{y}\left(t_{k}\right)<0, k=0, \ldots, N
\end{gathered}
$$

Concept 2 [8], [9], [27]. It is assumed that the ground reaction forces can be equivalently represented by two normal forces $F_{y A}, F_{y B}$ applied at end points $\mathrm{A}$ and $\mathrm{B}$ of the foot, with a horizontal force acting in the sole, as shown in Fig. 2 (b). Thus, the conditions that should be satisfied are

$$
\begin{gathered}
-F_{y A}\left(t_{k}\right)<0, k=0, \ldots, N \\
-F_{y B}\left(t_{k}\right)<0, k=0, \ldots, N \\
-F_{x}\left(t_{k}\right)-\mu\left(F_{y A}\left(t_{k}\right)+F_{y B}\left(t_{k}\right)\right)<0, k=0, \ldots, N \\
F_{x}\left(t_{k}\right)-\mu\left(F_{y A}\left(t_{k}\right)+F_{y B}\left(t_{k}\right)\right)<0, k=0, \ldots, N
\end{gathered}
$$

3) Bounded constraints

$$
\begin{aligned}
& \boldsymbol{q}_{\text {min }} \leq \boldsymbol{q}\left(t_{k}\right) \leq \boldsymbol{q}_{\text {max }}, k=0, \ldots, N \\
& \dot{\boldsymbol{q}}_{\text {min }} \leq \dot{\boldsymbol{q}}\left(t_{k}\right) \leq \dot{\boldsymbol{q}}_{\text {max }}, k=0 \text { and } N
\end{aligned}
$$

- Performance index (objective function)

In general, there are miscellaneous performance indices that can be used depending on the aim of the designer. If the minimum energy is required, the literature prefers the actuating torque cost on the energetic cost due to the instability problems of the latter. If the designer intends to focus on the best stability of the biped mechanism, the minimum deviation of ZMP can be used as cost function [1]. The latter reference has used combined cost function of the sum of the deviation of ZMP and the minimum energy. However, it can simply be dealt with ZMP as a constraint and deals with the minimum energy as cost function.

Anyway, we chose the actuating torques as a cost function to be minimized.

$$
J=\int_{t 0}^{t f} \boldsymbol{u}^{T} \boldsymbol{u} d t=\sum_{k=0}^{N-1} \boldsymbol{u}_{k}^{T} \boldsymbol{u}_{k} \cdot \Delta t
$$

\section{SimUlation RESUlts AND DisCUSSION}

After converting the dynamic optimization into parameter optimization, any NLP algorithm can be used. Most literatures [4], [8], [18] have used local solver represented by fmincon routine for the solution of the parameter optimization. It should be noted that this command needs good initial guess to capture feasible local minimum. We tried to use an arbitrary initial guess, but no feasible solution can be obtained. However, the inverted-pendulum based gait can provide us very good initial guess. Therefore, we depended on this strategy in all investigated cases [31]. One of the disadvantages of the fmincon is that it can get stuck in

\begin{tabular}{|c|c|c|}
\hline $\begin{array}{l}\text { Case } \\
\text { No. }\end{array}$ & Description & $\begin{array}{l}\text { Performance index } \\
\qquad\left(N^{2} \cdot m^{2} \cdot s\right)\end{array}$ \\
\hline 1 & $\begin{array}{l}\text { The biped robot is subjected to the } \\
\text { constraints (35) to }(45) \text {, with foot } \\
\text { rotation. }\end{array}$ & 14.21 \\
\hline 2 & $\begin{array}{l}\text { The same constraints of case } 1 \text { but } \\
\text { with foot level to the ground. }\end{array}$ & 57.32 \\
\hline 3 & $\begin{array}{l}\text { The same constraints of case } 1 \text { with } \\
\text { constant hip height. }\end{array}$ & 64.37 \\
\hline 4 & $\begin{array}{l}\text { The same constraints of case } 1 \text { with } \\
\text { lower hip height. }\end{array}$ & 144.7 \\
\hline 5 & $\begin{array}{l}\text { The same constraints of case } 1 \text { with } \\
\mathrm{L} \text { thigh }=1.25 \mathrm{~L} \text { shank. }\end{array}$ & 12.56 \\
\hline 6 & $\begin{array}{l}\text { The same constraints of case } 1 \text { with } \\
\mathrm{L} \text { shank }=1.25 \mathrm{~L} \text { thigh. }\end{array}$ & 16.05 \\
\hline 7 & $\begin{array}{l}\text { The same constraints of case } 1 \text { with } \\
\text { longer steps. }\end{array}$ & $\begin{array}{l}15.05(\text { Lstep }=0.5 \mathrm{~m}) \\
16.49(\text { Lstep }=0.6 \mathrm{~m})\end{array}$ \\
\hline
\end{tabular}
local minimum, therefore, hybrid genetic-sequential quadratic programming (ga-fmincon) was used to capture the global minimum, as shown in Fig. 3. However, we got the same minimum point resulted by the local solver. For details about the hybrid genetic- SQP we refer to [21], [32][34].

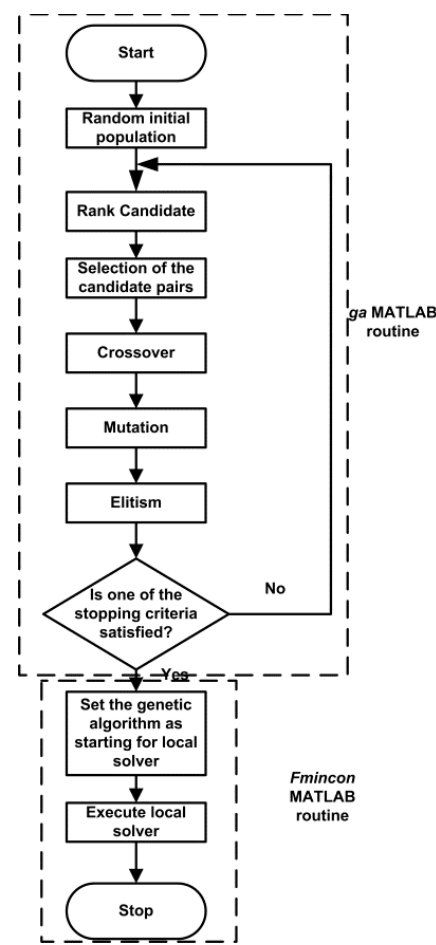

Fig. 3. Flow chart of hybrid genetic-local solver.

TABLE III: THE STUDIED CASES FOR THE SIMULATION

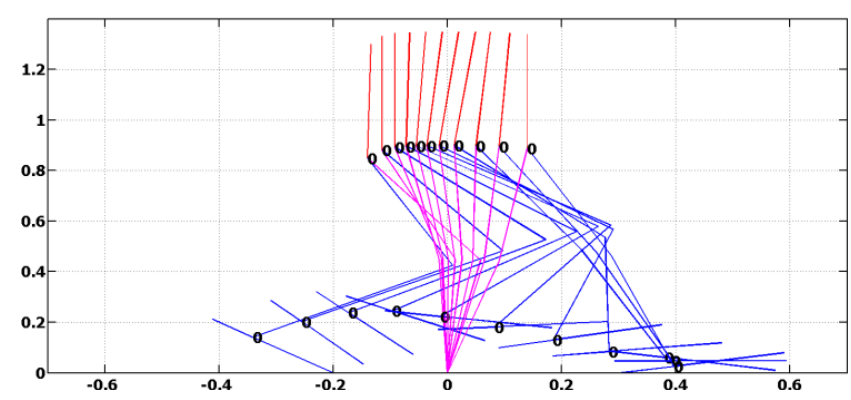

Fig. 4. Biped robot configuration for case 1.

Seven simulated cases were investigated for different purposes. Case 1 studied the optimal motion of biped robot subjected to the constraints and objective function described in the previous section, as shown in Fig. 4. Whereas in case 2 , the swing foot was restricted to be always level to the ground, as shown in Fig. 5. From Table III, it can be noticed that case 2 needs more performance index than that of case 1 
due to the imposed constraint. For the next cases (case 3-7), we considered case 1 as reference for the purpose of comparison. In cases 3 and cases 4, more energy is needed if the biped robot constrained to move with constant hip or lower hip height. The more imposed constraints, the more energy are demanded.

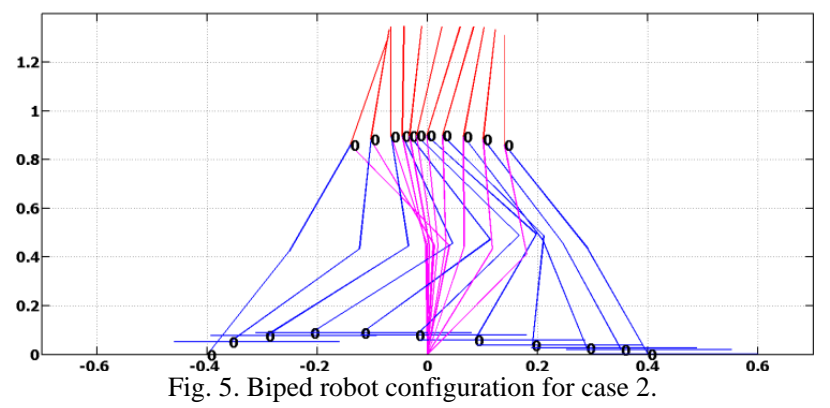

On the other hand, the effect of the thigh and shank length were studied via cases 5 and 6 by keeping the total length of the leg fixed and changing the proportion of the thigh and the shank. The longer thighs the slightly less energy can be needed. Whereas, the increase in the required energy is slight if the shank length is longer than the thigh length. The last case included the effect of increase of the step length. The longer steps the slightly more actuating torques are demanded. The time elapsed in all cased was taken as $0.5 \mathrm{~s}$ while the step length was assumed equal to $0.4 \mathrm{~m}$ in cases 1 6.

\section{CONCLUSIONS}

This paper focuses on three issues: the selection of the suitable direct method for suboptimal control of the biped robot, the suitable algorithm for the solution of the global minimum of the NLP, and the effect of different constraints on the required energy of the biped robot. The finite difference approach can be used efficiently to solve the suboptimal trajectory of the biped mechanism. While the genetic-SQP can get the global minimum of the NLP. Lastly, it can be concluded that more imposed constraints on the biped robot, more energy is needed. In general, more energy can be required in the case of

- Restriction of the swing foot to be level to the ground.

- Reducing the hip height or constraining the hip to move in constant height.

- Longer shanks than thighs.

- Longer steps or high step speed.

In effect, a slight increase of the energy was produced in the last two cases. Despite the easy use of the suboptimal control problem, it can give approximate solution. Therefore, further study is necessary to deal with the problem in real time application.

\section{REFERENCES}

[1] P. R. Vundavilli and D. K. Pratihar, "Gait Planning of Biped Robots Using Soft Computing: An Attempt to Incorporate Intelligence," in Intelligent Autonomous Systems: Foundation and Applications, D. K. Pratihar and L. C. Jain, Ed., Germany: Springer-Verlag, 2010, ch. 4, pp. 57-85.

[2] H. F. N. Al-Shuka, F. Allmendinger, and B. Corves, Modeling, stability and walking pattern generators of biped robots: A historical perspective.
[3] S. Kajita and K. Tani, "Experimental study of biped dynamic walking in the linear inverted pendulum mode," in Proc. IEEE Conf. Robotics and Automation, 1995, vol. 3, pp. 2885-2891.

[4] C. Chevallereau, G. Bessonnet, G. Abba, and Y. Aoustin, Bipedal Robots, Modeling, Design and Building Walking Robots, $1^{\text {st }}$ ed.,U.K.: John Wiley and Sons Inc ,2009,ch. 4,pp. 219-265.

[5] R. D. Robinett III, D. G. Wilson, G. R. Eisler, and H. E. Hurtado, "Applied dynamic programming for optimization of dynamical systems," SIAM, Philadelphia, USA, 2005.

[6] M. G. Pandy, F. C. Anderson, and D. G. Hull, "A parameter optimization approach for the optimal control of large-scale musculoskeletal," J. Biomech. Eng., vol. 114, no. 4, pp. 450-460, 1992.

[7] M. Diehl, "Numerical optimal control," Lecture notes, Optimization in Engineering Center (OPTEC) and Electrical Engineering Department (ESAT), K. V. Leuven, Belgium, 2011.

[8] P. Seguin and Bessonnet, "Generating optimal walking cycles using spline-base state parameterization," International Journal of Humanoid Robotics, vol. 2, no. 1, pp. 47-80, 2005.

[9] M. Rostami and G. Bessonnet, "Sagittal gait of a biped robot during the single support phase. Part 2: Optimal motion," Robotica, vol. 19, pp. 241-253, 2001

[10] G. Bessonnet, S. Chesse, and P. Sardain," Generating optimal gait of a human-sized biped robot," in Proc. the $5^{\text {th }}$ Int. Conf. Climbing and Walking Robots, Paris, 2002, pp. 241-253.

[11] D. G. Hull, "Conversion of optimal control problems into parameter optimization problems," AIAA, Guidance, Navigation and control performance, San Diego, 1996.

[12] C. J. Goh and K. L. Teo, "Control parameterization, A unified approach to optimal problem with general constraints," Automatica, vol. 24. no. 1, pp. 3-18, 1988.

[13] B. Chachuat, "Optimal control, Lectures 19-20: Direct solution methods," Department of chemical engineering, McMaster University, Canada, 2009.

[14] S. C. Chapra, Applied numerical methods with MATLAB ${ }^{\circledR}$ for engineers and scientists, New York, USA: McGraw Hill Higher Education, 2008

[15] V. M. Becerra, "Practical direct collocation methods for computational optimal control," in Modeling and optimization in space engineering, G. Fasano and J. D. Pinter, Eds., Springer Optimization and Its Applications, 2013.

[16] O. von Stryk, "Numerical solution of optimal control problems by direct collocation," in Optimal control-Calculus of variations, optimal control theory and numerical methods, R. Bulrisch, A. Miele, J. Stoer, and K.-H. Well, Eds., International Series of Numerical Mathematics, pp. 129-143, 1993.

[17] J. T. Betts, "Practical methods of optimal control using nonlinear programming," SIAM, Philadelphia, USA, 2001.

[18] C. Azevedo, P. Poignet, and B. Espiau, "On line optimal control for biped robot," 15 th Teriennial World Congress, Barcelona, Spain, 2002.

[19] L. Roussel, C. Canudas-de-Wit, and A. Goswami, "Generation of energy optimal complete gait cycles for bipedal robot," in Proc. Conference on Robotics and Automation, IEEE, vol. 3, pp. 2036-2041, 1998.

[20] Optimization Toolbox ${ }^{\mathrm{TM}}$, User's Guide, The MathWorks, Inc., 2011.

[21] Global Optimization Toolbox ${ }^{\mathrm{TM}}$, User's Guide, The MathWorks, Inc., 2011.

[22] A. Hamon and Y. Aoustin, "Cross four-bar linkage for the knees of a planar bipedal robot," in Proc. IEEE-RAS International Conference on Humanoid Robots, 2010, pp.379-384.

[23] S. Tzafestas, M. Raibert, and C. Tzafestas, "Robust sliding mode control applied to 5-link biped robot," Journal of Intelligent and Robotic Systems, vol. 15, pp. 67-133, 1996.

[24] L. Roussel, C. Canudas-de-Wit, and A. Goswami, "Comparative study of methods for energy-optimal gait generation for biped robots," International Conference on Informatics and Control, 1997.

[25] V. Yen and M. L. Nagurka, "Suboptimal trajectory planning of a fivelink human locomotion model," Biomechanics of normal and prosthetic gait, ASME, BED-vol. 4 and DSC-vol. 7, pp. 17-22, 1987.

[26] C.-S. Lin, P.-R. Chang, and J. Y. S. Luh, "Formulation and optimization of cubic polynomial trajectories for industrial robots," IEEE Transactions on automatic control, vol. AC-28, no. 12, pp. 1066-1074, 1983.

[27] T. Saidouni and G. Bessonnet, "Generating globally optimised sagittal gait of a biped robot," Robotica, vol. 21, pp. 199-210,2003.

[28] Q. Wang, "A study of alternative formulations for optimization of structural and mechanical systems subjected to static and dynamic loads," PhD Dissertation, University of IOWA, USA, 2006. 
[29] Q. Wang and J. S. Arora, "Alternative formulations for transient dynamic response optimization," AIAA, vol. 43, no. 10, pp. 21882195, 2005.

[30] B. Vanderborght, "Dynamic stabilization of the biped Lucy powered by actuators with controllable stiffness," $\mathrm{PhD}$ dissertation, Vrije Universiteit Brussel, Belgium, 2007.

[31] H. F. N. Al-Shuka and B. J. Corves, "On the walking Pattern generators of biped robot," Journal of Automation and Control Engineering, vol. 1, no. 2, pp.149-155, 2013.

[32] N. Yokoyama and S. Suzuki, "Flight trajectory optimization using genetic algorithm combined with gradient method," ITEM, vol. 1, no. $1,2001$.

[33] F. Yengui, L. Labrak, F. Frantz, R. Daviot, N. Abouchi, and I. O'Connor, "Hybrid GA-SQP algorithm for analog circuits sizing," Circuits and Systems, vol. 3, pp. 146-152, 2012.

[34] K. Okuda, K. Yonemoto, and T. Akiyama, "Hybrid optimal trajectory generation using genetic algorithm and sequential quadratic programming," ICROS-SICE International Joint Conference, Japan, 2009.

Hayder F. N. Al-Shuka was born in Baghdad, Iraq in 1979. He received the B.Sc and M.Sc. degrees from Baghdad and Al-Mustansiriya
Universities respectively, Baghdad, Iraq, in 2003 and 2006 respectively. Since 2006, he has been assigned as an assistant lecturer in Baghdad University at the department of Mechanical Engineering. He is currently $\mathrm{PhD}$ student at the RWTH Aachen University at the Department of Mechanism and Machine Dynamics. His research interests include the walking patterns and control of biped robots.

Burkhard J. Corves was born in Kiel, Germany in 1960. He received the Diploma and PhD degrees in Mechanical Engineering from RWTH Aachen University, Aachen, Germany, in 1984 and 1989 respectively. From 1991 until 2000, he got teaching assignment in RWTH Aachen University. Since 2000, he has been appointed as university professor and director of the department of Mechanism and Machine Dynamics of RWTH Aachen University. The research interests of Prof. Dr. Corves include the kinematics and dynamics of mechanisms and robots.

W.-H. Zhu is an engineering technical officer at the Canadian Space Agency. His specialty is on precision control of complex robotic system characterized by his book entitled Virtual Decomposition Control published by Springer-Verlag in its STAR series. Dr. Zhu also published $60+$ papers in leading international journals and conference proceedings. 